\title{
ATIOLOGY AND PATHOGENESIS OF ACUTE SPORADIC DISSEMINATED ENCEPHALOMYELITIS AND MULTIPLE SCLEROSIS
}

\author{
BY
}

\author{
M. S. MARGULIS, V. D. SOLOVIEV, and A. K. SHUBLADZE \\ (From the Central Institute of Microbiology and Epidemiology, and the Clinic of Nervous Diseases attached to the \\ Postgraduate Institute for Physicians, the Botkin Hospital, Moscow, U.S.S.R.)
}

(RECEIVED 24TH APRIL, 1946)

THE investigation of nervous diseases caused by viruses requires close co-operation between clinical and experimental workers. The present paper is the summary of such co-operative work performed by a neuro-histologist and a team of workers in virus diseases. Its chief purpose is to throw some light upon the ætiology and pathogenesis of acute disseminated encephalomyelitis and multiple sclerosis, because up to now many unsolved problems remain, not only in the field of ætiology but also in that of their morphology and pathogenesis. Some authors are still inclined to doubt the infectious origin of multiple sclerosis, and offer a number of theories attempting to explain the pathogenesis of this disease, such as Putnam's obstruction theory, and toxic, allergic, lipolytic, and other theories. We shall not dwell on the non-infectious hypothesis of multiple sclerosis, because the authors advocating this theory take the effect of the pathological process for its cause. These hypotheses are based on changes occurring in individual morphological elements (glia) or on individual normal or abnormal responses of the organism, and so fail to yield an outline of the whole pathological process; they thus cannot give a true idea of pathogenesis.

The result of virus and post-mortem investigation of many patients suffering from acute disseminated encephalomyelitis and multiple sclerosis was that two strains of filtrable viruses were isolated from two cases of acute disseminated encephalomyelitis. These strains appeared to be identical, but differed from other known strains of neurotropic viruses encountered in man.

\section{Case Histories}

The case histories of these patients are as follows:

Case 1.-Sv., aged 32, electrician. Entered the Clinic on Nov. 18, 1942. Fell ill Nov. 17, 1942. He suddenly felt his lower limbs heavy and the next day was unable to walk without help. In the evening he developed paralysis of the lower extremities. The temperature on admission was $37.6^{\circ} \mathrm{C}$. Examination revealed clear consciousness. There was lateral nystagmus, but no other abnormality of the cranial nerves; paralysis of the lower extremities, with ankle clonus, increased tendon jerks, yet absence of pathological reflexes was found; hypæsthesia in the distribution of segment $L .2$ and anæsthesia in that of segment L.3 were present. The patient complained of headache and dizziness. Albino mice were inoculated with the patient's blood and cerebrospinal fluid. A virus was isolated from the blood after the second passage.
Nov. 24: The patient was listless and drowsy. He awoke only to take his meals. He was able to perform small movements with his lower extremities. His fundi were normal. Nov. 30: His temperature rose to $37 \cdot 8^{\circ} \mathrm{C}$. in the evening. Dec. 1: His temperature was $37.5^{\circ}-$ $37.9^{\circ} \mathrm{C}$. Weakness in the distal part of the upper extremities developed. Impairment of superficial sensation of both hands of root type was found. Power of movement was retained only at the knee joints. The patient showed the same disturbances of sensation as on admission. He also exhibited mild meningeal symptoms. Dec. 3: The temperature was normal, the patient felt better. The range of movements in the lower extremities had increased and the upper limit of anæsthesia was lower. A slightly positive Laségue sign was found. The patient complained of pains in his feet. Dec. 21 : The cerebrospinal fluid was clear and colourless with 0.03 per cent. albumin and no cells. The Wassermann reaction was negative both in blood and cerebrospinal fluid. Jan. 13, 1943: Increase in the extent of movements in the lower extremities was found. Bilateral Laségue signs: tenderness of nerve trunks on pressure in all the 4 extremities were present. There was improvement of sensation. Jan. 16: The patient was able to rise without assistance from his bed. Impairment of sensitivity in the lower extremities was of distal type with a decrease in sensory loss in the hands. Feb. 11: His temperature was $36 \cdot 5^{\circ}-37 \cdot 2^{\circ} \mathrm{C}$. and his condition had deteriorated. Consciousness was dulled. The pulse rate was 90 . A rotatory nystagmus and flattened left nasolabial fold were observed. Pressure on the nerve trunks was painful. There was retention of urine and a right-sided Sabinski response was obtained. Feb. 17: The patient's condition showed some deterioration. Nystagmus and retention of urine were still present. Feb. 24: Further deterioration in the patient's condition had taken place. In the evening he exhibited motor excitement: he collected all his belongings, tore off an electric wire from the wall and put it under the table, then made for the exit, pushing aside the hospital personnel and declaring that he must join his mother. March 2: Some amelioration in the patient's condition was noticed. Objectively there was absence of meningeal symptoms and of abnormal reflexes. Subjectively he complained of bilateral tenderness of nerve trunks. Subsequently the patient continued to show gradual improvement, but some degree of tenderness of nerve trunks together with slight retention of urine remained. These symptoms eventually subsided and he was discharged symptom-free on April 22, 1943. The clinical diagnosis of acute disseminated encephalomyelitis was made.

The patient was again admitted to the Clinic on June 28, 1944, because of an exacerbation of his disease. On admission he showed drowsiness, lateral nystagmus, slight paresis of his left limbs, and unsustained ankle clonus. Rossolimo's sign and an indefinite Babinski sign were present on the right side with hemihypæsthesia to all forms of sensation on the left. Some muscular rigidity in the occipital region and bilateral Kernig's signs were found. The cerebrospinal fluid was clear, with a 
normal pressure; there were 4 cells and 0.03 per cent. albumin. Power gradually returned in the left limbs; the hypæsthesia and meningeal symptoms subsided. On Aug. 1 the patient was discharged showing a satisfactory condition and an absence of objective symptoms of functional failure. The patient again entered the Clinic on March 22, 1945, complaining of headache and drowsiness. He answered questions in a listless way and fell asleep during conversation. He was disoriented and showed mental inhibitions and stupor; his face was expressionless; at times he was restless. He had nystagmus to the left; there was a lack of convergence; he had a slow speech with paresis of the left limbs. Tendon reflexes were more active on the left side, with Babinski and Rossolimo signs on that side, while Rossolimo, Bendel-Bechterev, and Jukovski signs were found on the right side. There was some ataxia. He was unable to stand and to walk. Bilateral Kernig and Brudinski signs were noted. There appeared to be a degree of hyperpathia involving the left side of the body. Joint sense was lost in the fingers and toes. The patient's condition improved considerably a month later, by which time he could walk and live a normal life. The Babinski sign disappeared, though that of Rossolimo still remained. $\mathrm{He}$ was discharged on June 28,1945 , in a satisfactory condition and continued a course of vaccine-therapy in the outpatient department. His condition is still quite good at present.

Case 2.-Ef., woman, aged 38. Entered the Clinic on Feb. 14, 1943. Her complaints were: general weakness, pains in the chest, headache, sensation of weakness in the extremities, and inability to walk. The patient had had an attack of influenza in 1942, with a temperature of $39^{\circ} \mathrm{C}$. She had been ill and away from work for some days. She resumed her work, but on Jan. 15, 1943 , her temperature rose to $38^{\circ}-39^{\circ} \mathrm{C}$. and her physician thought she had pneumonia.

On admission the patient was listless, her face was expressionless, her mentality was slow, and her sleep poor. She was inattentive and readily fatigued. The sight of the left eye was misty. Her pupillary reflex to light was satisfactory, but convergence was poor; the movements of the eyeball were normal. The visual acuity of the left eye was considerably lower than that of the right. The fundi were normal. There was no abnormality in the other cranial nerves. There was slight neck stiffness; power was normal in the arms and legs. There was increased muscular tone, especially on the left side; the tendon jerks were brisk, with left-sided Babinski's sign; the abdominal reflexes were absent; no changes in superficial and deep sensitivity were elicited. There was a defect of posture with intention tremor in both hands in the finger-nose test. Feb. 16: Her temperature was $36 \cdot 3^{\circ}-37 \cdot 3^{\circ} \mathrm{C}$. Lumbar puncture showed clear fluid under increased pressure. Blood and cerebrospinal fluid were collecter in order to carry out virus examination. Feb. 17: Her temperature was $36.6^{\circ}-$ $37 \cdot 4^{\circ} \mathrm{C}$. The patient's condition had improved and she was more alert. She was sleepless and had a pulse rate of 70 ; there was no paralysis of extremities, yet there was increased tendon jerks, with bilateral ankle clonus and an extensor plantar response on the left side.

Blood examination revealed: leucocytes, 12,200 per 100 c. mm. of blood; segmented neutrophils, 29 per cent.; stab cells, 32.5 per cent.; eosinophils, 0 per cent.; lymphocytes, 15 per cent.; monocytes, 2 per cent.; immature forms, 1 per cent.; Turck cells, 0.5 per cent.; Wassermann reaction, negative. The cerebrospinal fluid was clear and colourless, with no cells and 0.09 per cent. albumin; cerebrospinal fluid Wassermann reaction was negative. The other organs were normal; blood pressure was $100 \mathrm{~mm}$. Hg. systolic and 60 diastolic. Feb. 19: Her temperature was $36 \cdot 3^{\circ}-35 \cdot 6^{\circ} \mathrm{C}$. The condition of the patient was poor with increased salivation, clonus of both feet, and slight neck rigidity; Kernig's sign was present. Feb. 20: Temperature was normal. The patient had a restless night, being excited and crying for help. She imagined that she was falling, that she was being carried away somewhere, and that she was contaminated with some disease. At this time she failed to show the changes in her reflexes and in her motor and sensory systems as compared with her previous examination. Feb. 22: Her condition was serious. The patient showed no reaction to her environment; her tendon reflexes were decreased. Heart sounds were inaudible and she died the same day at $8 \mathrm{p} . \mathrm{m}$. from heart failure. The clinical diagnosis of acute disseminated encephalomyelitis was made.

An autopsy was performed by Prof. Margulis on Feb. 23, 1943. No changes were found in the lungs; the heart muscle was flaccid with enlarged ventricular cavities, but no abnormality of the valves; the stomach was normal; a catarrhal condition of the intestinal mucous membrane was present; no gross changes apart from some congestion of the meninges and cerebral substance were found in the brain.

Microscopy revealed scattered inflammatory vascular foci in the white substance of the hemispheres, the brain stem, and the medulla oblongata. By means of myelin stains, scattered necrotic foci were detected in the brain and brain stem. Around some of these foci a more or less intense inflammatory vascular reaction was encountered. Proliferation of microglia and of astrocytic glia was a constant occurrence in the immediate neighbourhood of these foci, without showing much formation of glial fibres. Disseminated foci of demyelination and segmented demyelination of nerve fibres were encountered, together with miliary necrosis.

From the histopathological viewpoint this case proved identical with the other of primary acute disseminated encephalomyelitis.

\section{Experimental Investigations}

Albino mice were inoculated with the cerebral subo stance obtained at autopsy from Case 2. The virus was isolated after the first passage. In our first clinical case of encephalomyelitis, the pathogenic agent was isolate from the blood of the patient and called "Sv." strain; i⿱⺈. the second case the agent was isolated from cerebrat tissue and called "Ef," strain. We have adopted these terms and shall use them later. Albino mice, puppies, rats, guinea-pigs, and rabbits were inoculated with both strains.

As the result of histo-pathological examination of these animals we found that, apart from considerable congestion, no gross changes were demonstrable in the brain. Microscopy, however, revealed infiltration with lymphocytes of the pia both in brain and spinal cord; hæmorrhage around vessels and between the laminæ of the pia was noted. Inflammatory foci in the brain and spinal cord around the vessels were made up of adventitial cells and proliferating microglia; numerous perivascular hæmorrhages were also present; granulomata formed of microglial cells and hystiocytes derived from vascular walls were encountered in the neighbourhood of perivascular cuffs. The inflammatory changes occurred more frequently in the white substance of the brain whereas in the spinal cord no preponderance of inflammatory changes in the white substance was noted. Brains and spinal cords of puppies and rabbits, when stained by Spielmeyer's method, disclosed miliary necrosis in the nervous tissue, scattered throughout the central nervous system. Alongside these minute necrotic foci, focal and fascicular demyelination of nerve fibres was detected.

Proliferation of microglia was seen around the necrotic and demyelinated zones. Fibrous astrocytes were involved in the proliferative process developing around the necrotic foci. At places where necrobiosis of the nervous tissue had occurred, as revealed by the Snessarev stain, the development of argyrophil fibres was demonstrable. As a result of this proliferation, formation of plaques occurred around necrotic foci. Microglia, macroglia, and argyrophylic fibres formed the basis of these plaques, which occasionally took the stains for collagen. These plaques closely resembled those observed in acute disseminated encephalomyelitis and 


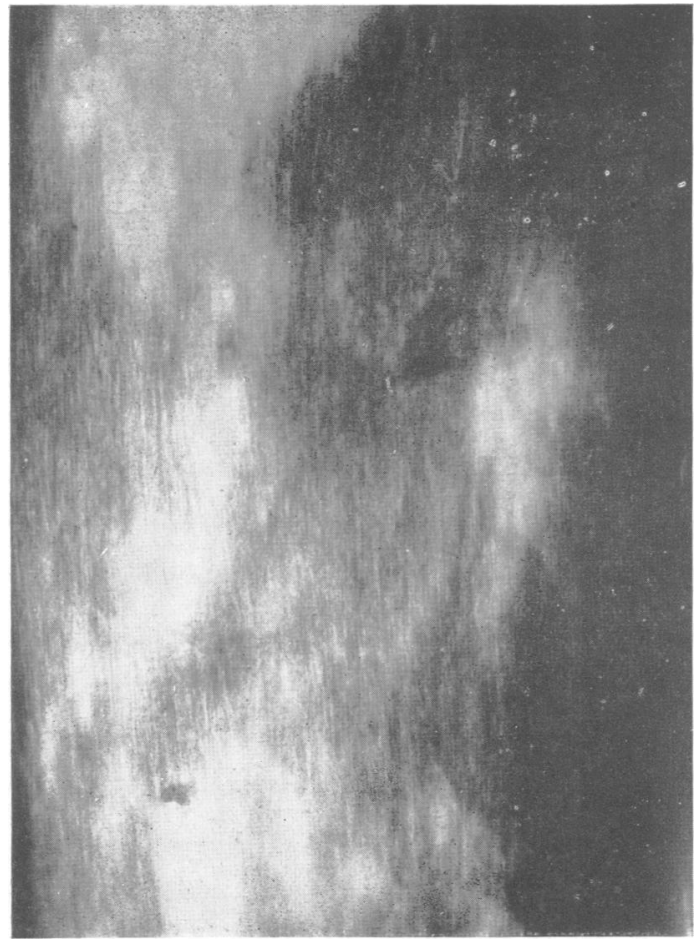

FIG. 1.-Experimental "Sv." encephalomyelitis. Rabbit (N23). Stained after Spielmeyer. Brain. Demyelination.

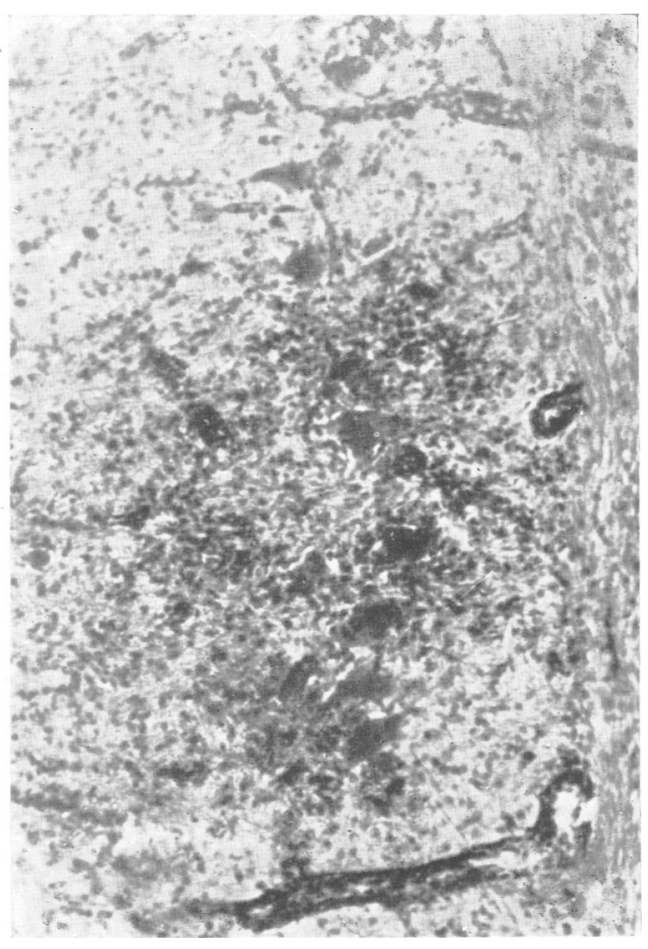

FIG. 2.-Experimental " Sv." encephalomyelitis. Puppy. Stained with thionine. Cells of the anterior horns. Neuronophagia. multiple sclerosis. When stained by the Bielschowski method, these plaques were found to contain a considerable number of preserved axis cylinders, together with degenerated and fragmented remains. In addition to perivascular proliferation of the microglia, focal proliferation in the shape of nodules was also encountered in the grey and white substances of both brain and spinal cord. Neuronophagia around nerve cells, mostly in the spinal cord, was less frequently seen in rabbits than in puppies. The nerve cells exhibited various stages of necrobiosis and were surrounded by numerous satellite cells. As a result of necrobiosis and neuronophagia the nerve cells disappeared, leaving in their place "nodules" made up of microglia. Neuronophagia was also seen in the cortex, but was less pronounced and occurred only occasionally in the form of small foci. Around the proliferative nodules in the cerebral white substance of puppies, rabbits, and guinea-pigs there were large numbers of microglial cells which showed a gradual transition into the normal tissue without a definite border. Most of the microglial elements in this proliferative area exhibited various stages of degeneration.

\section{Analysis of Experiments}

The analysis of the morphological changes found in experimental encephalomyelitis produced in animals with the "Sv." and "Ef." viruses revealed that the changes differed from those of herpes encephalitis; demyelination is absent in herpes encephalitis. Despite numerous investigations of paraffin sections stained with Heidenhain's hæmotoxylin or with methylene blue after Mabb, we failed to detect any kind of inclusions either in nerve cells, in vascular endothelium, or in pia; on the other hand, control preparations from animals suffering from herpes encephalitis and stained in a similar manner, showed specific intranuclear inclusion bodies. The presence of demyelination also contributed to the differential diagnosis between experimental disseminated encephalomyelitis in animals inoculated with the "Sv." virus and focal hæmorrhagic encephalitis. The group of animals showing susceptibility to focal encephalitis is confined to albino mice; we succeeded, however, in contaminating rabbits under special conditions, and in these animals no demyelination was demonstrable.

Small necrotic foci and zones of demyelination were the earliest changes in the central nervous system in the animal infected with "Sv." and "Ef." virus. As the virus ætiology of miliary necrosis occurring in experimental encephalomyelitis brought about by the "Sv." virus is beyond doubt, there is reason to suggest an infectious origin of analogous miliary necroses as found in disseminated acute encephalomyelitis and multiple sclerosis. Miliary necrosis is the necrobiotic reaction of the nervous tissue; it is unrelated to vascular changes, and arises during the initial contact of the "Sv." virus and the nervous tissue. Miliary necrosis is not pathognomonic for any kind of virus; it is encountered in various virus infections of the nervous system, accompanied or not by demyelination. Hence, miliary necrosis is to be considered as a non-specific and non-differentiated response of the nervous tissue to the invasion of virus agents, which obeys the general laws of pathology. Another constant morphological finding in experimental encephalomyelitis ("Sv." virus), 
namely, demyelination (Fig. 1), frequently develops with proliferative and inflammatory changes around the blood vessels; but these processes are independent of demyelination. As with miliary necrosis, demyelination is not pathognomonic of encephalomyelitis. Demyelination should not be considered as necessarily a specific feature of infectious processes, for it is also encountered in various toxicoses, metabolic disturbances, and hæmatological diseases. Nevertheless, without being pathognomic for any individual form of infection, demyelination is a characteristic feature of a group of demyelinating encephalomyelitis. Some species, when inoculated with the "Sv." and "Ef." viruses (for instance, puppies and rabbits) constantly exhibited demyelination of nerve fibres, whereas other animals, such as guinea-pigs and albino rats, showed slight and limited demyelination, although all these animals were inoculated with the same virus. Apparently, limited demyelination may be accounted for by a particular sensitivity of the nervous tissue to the "Sv." and "Ef." viruses, which are fixed in certain sites of the nervous tissues as soon as they invade the tissue. As a result of this fixation, new conditions of metabolism within the tissues arise at these sites, thereby favouring demyelination. Hence it occurs in nervous tissue under special and peculiar conditions which still remain obscure. The atrophic degenerative changes found in the cortical nerve cells, in the anterior horn and lateral horn cells of the spinal cord both in puppies and rabbits, were accompanied by neuronophagia (Fig. 2). Neuronophagia was well marked in puppies and much less in rabbits. But in other animals we failed to detect neuronophagia even though atrophic degenerative changes in nerve cells were present in all the experimental animals. Hence necrobiosis alone may be unable to call forth neuronophagia. Necrobiosis and neuronophagia are therefore not necessarily concomitant processes. The absence of neuronophagia might be accounted for by metabolic conditions in the nervous tissue or by formation of metabolic products and enzymes inhibiting the satellite reaction.

The inflammatory vascular reaction forms one of the main components in the morphological picture of encephalomyelitis in animals caused by inoculation of the "Sv." virus. This inflammatory vascular reaction is probably a secondary process arising as a response to the primary necrobiotic changes in the nervous tissue caused by the virus. Variation in the intensity of the inflammatory vascular reaction will depend upon the reactivity of the nervous tissue and the species of the experimental animal. The proliferation of microglia and macroglia around miliary necroses, foci of demyelination and necrobiotic nerve cells, namely, around the sites where the virus primarily comes into contact with the nervous tissue, is evidence that proliferation of these elements occurs in response to the primary alteration of nervous tissue occurring during fixation of the virus. Apparently extensive microglial proliferation has the same functional significance, as evidenced by the presence of a large number of microglial elements, which have undergone degeneration under the influence of the virus. The development of argyrophylic fibres in the site of necrobiotic changes is evidence of the reconstructive role of mesenchymal tissue proliferation in these cases. (See Figs. 3-7.)

Both strains ("Sv." and "Ef.") were isolated by means of intracerebral passage of the initial material in albino mice. All the experiments were regularly checked by the inoculation of various artificial nutritive media. On the basis of absence of growth in such media and of positive results in animals obtained by inoculation of filtrates of the isolation strains, the infective agent was considered to belong to the group of filtrable viruses. Filtration experiments were carried out using membraneous filters, laminous Seitz filters, Berkefield V, and Chamberland $\mathrm{L}_{3}$ and $\mathrm{L}_{15}$ filters. For this experiment a 1 per cent. suspension of cerebral tissue collected from inoculated animals in meat-peptone broth of $p \mathrm{H} 7.4$ was used. In each filtration experiment a culture of B. prodigiosus was used as a control. The diameter of these organisms is known to be of $750 \mathrm{~mm}$. All the above-mentioned filters except the membraneous one retained the B. prodigiosus, but readily allowed the passage of the "Sv." and "Ef." strains. Both these strains may be kept in a 50 per cent. solution of glycerine, and after drying in a vacuum apparatus they may be preserved for a year and more.

Investigation of the effect of temperature on the "Sv." and "Ef." viruses yielded interesting dato. These strains showed stability to both cold tem perature and heating. They maintained their viability at a temperature of $20^{\circ} \mathrm{C}$. for a month, and at $37^{\circ} \mathrm{C}$. for 10 days. When heated to $60^{\circ} \mathrm{C}$. these viruses retained their activity for at least 60 minutes. Owing to this thermostability, they differ considerably from certain other viruses known for their thermolability.

Investigations directed to determine pathogenic features peculiar to the "Sv." and "Ef." virus showed that albino and grey mice, rats, guinea-pigs, rabbits, and young dogs exhibited susceptibility towards these strains. These animals were mostly sensitive to injections into the central nervous system. Inoculation, using the paraneural regions, also proved successful, but only with greater concentration of the virus. The inoculated animals all showed a similar clinical picture. After a latent period, the duration of which seldom exceeded 10 days, the mice usually showed tremor, transient clonic convulsions, and sometimes incessant movements of both hind and fore limbs, which made the animal rest on its side. Larger animals (belonging to other species) sometimes developed paralysis which usually occurred in the terminal stage of the disease. Of all the investigated animals, puppies, albino mice, and rabbits showed the greatest susceptibility to the virus.

For the purpose of serological identification of the isolated strains, neutralization experiments were performed with the serum of individuals who 


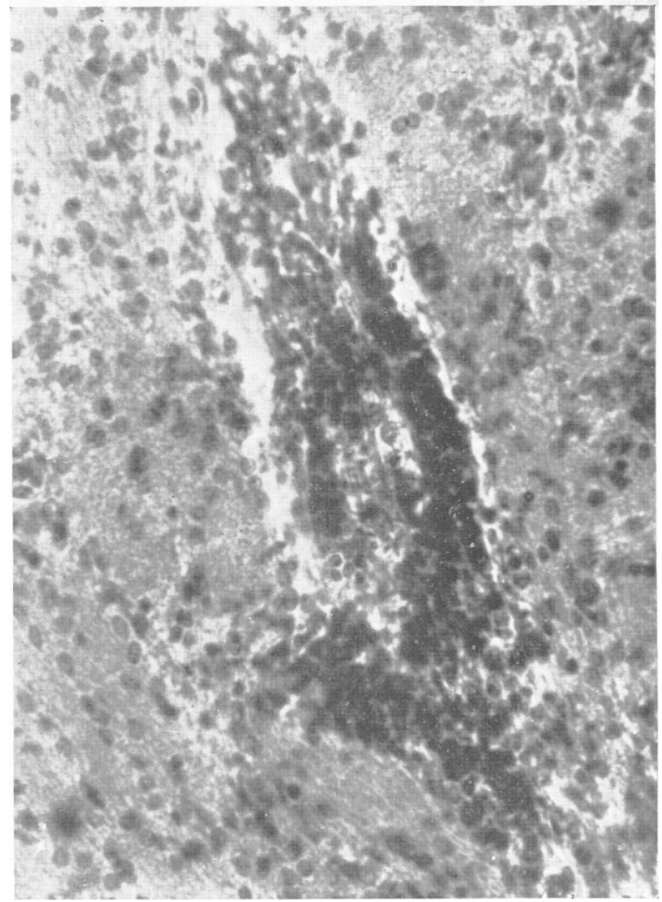

Fig. 3.

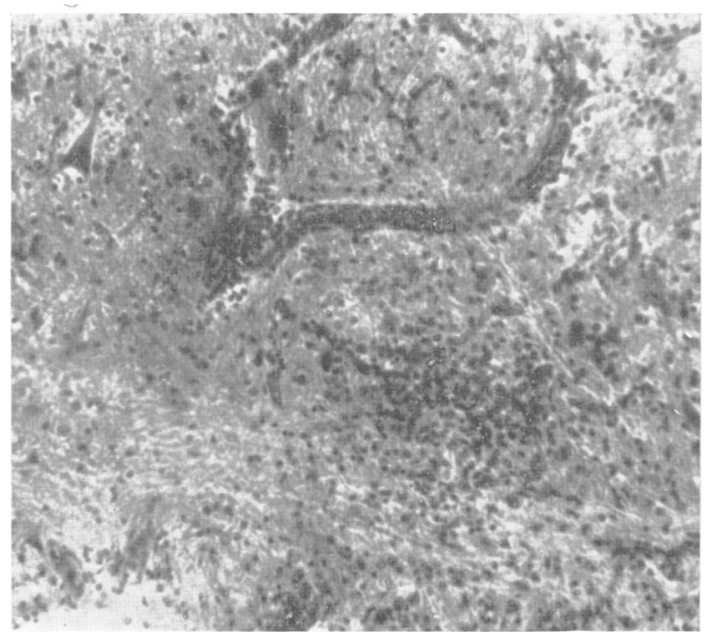

FIG. 5.

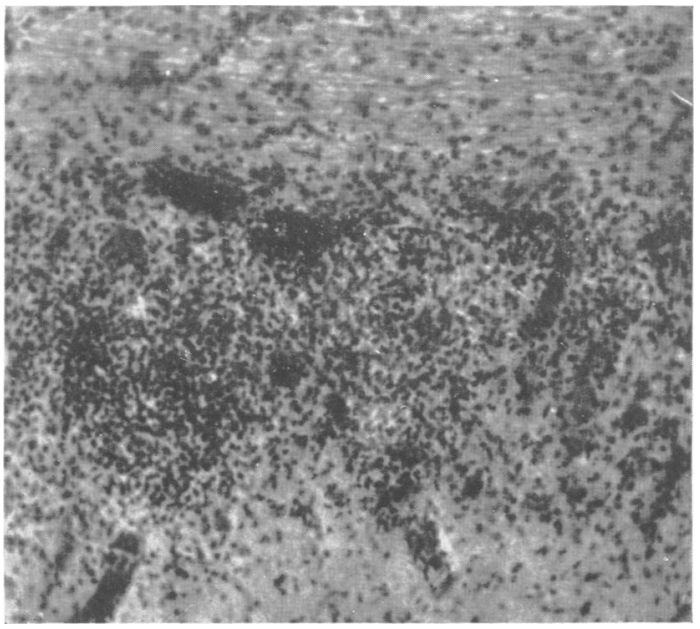

FIG. 6.

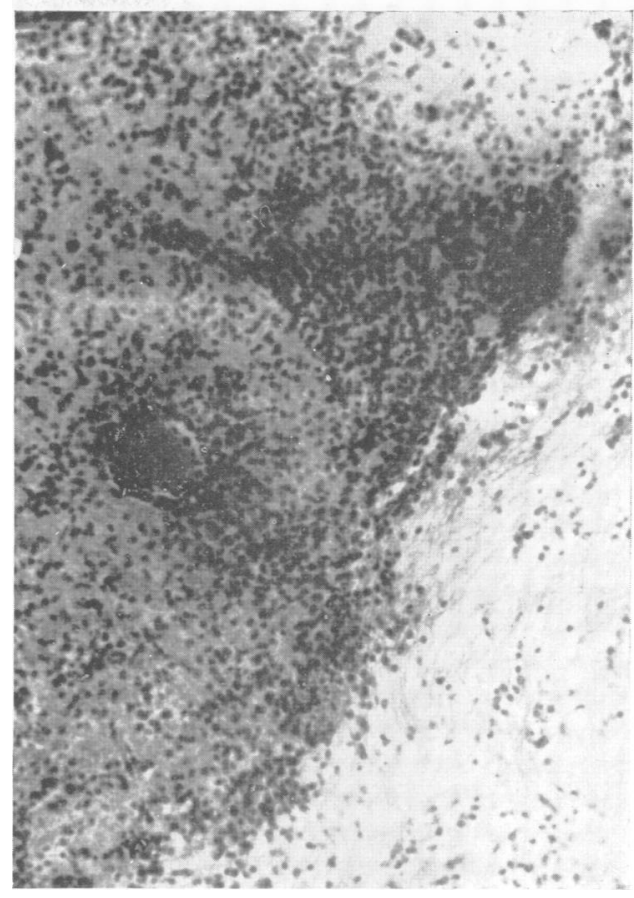

FIG. 4.

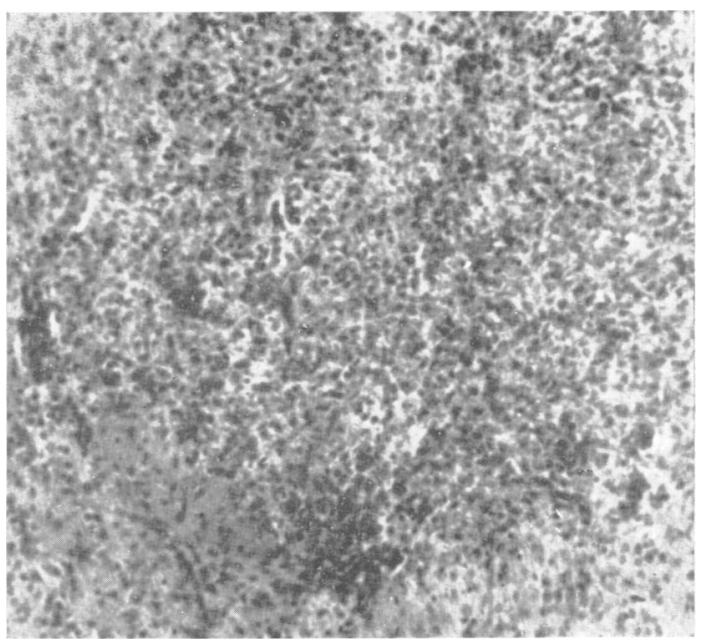

FIG. 7.

FIG. 3.-Experimental " Sv." encephalomyelitis. Puppy. Cellular infiltration of the adventitia of a blood vessel. Stained with hæmotoxylin-eosin.

FIG. 4.-Experimental "Sv." encephalomyelitis. Guineapig (N3). Cellular infiltration around blood vessels. Granulomata. Stained with hæmotoxylin-eosin.

Fig. 5.-Experimental "Sv." encephalomyelitis. Rabbit (N3). Cellular infiltration in the neighbourhood of a blood vessel in the grey substance of the spinal cord. Granuloma in the white substance. Stained with hæmotoxylin-eosin.

FIG. 6.-Experimental " Sv." encephalomyelitis. Rabbit (N4). Spinal cord. Stained with hæmotoxylin-eosin. Inflammatory vascular :eaction in the white substance. Proliferation of macroglia.

FIG. 7.-Exierimental " Sv." encephalomyelitis. Puppy. Stained with hæmotoxylin-eosin. Large microglial infiltration of the white substance of the medulla oblongata. 
had suffered acute disseminated encephalomyelitis. Serum was separated from the blood obtained from patients and mixed with equal volumes of various dilutions of virus. This mixture was incubated at a temperature of $37^{\circ} \mathrm{C}$. for 60 minutes, and, after this, mice were inoculated intracerebrally with $0.03 \mathrm{c} . \mathrm{cm}$. of this mixture. Each dilution of the virus-serum mixture was injected into 3 or 4 animals. The presence of virus-neutralizing antibodies in the serum was calculated on the basis of the difference between the number of mice who had developed the disease and controls. Similar doses of virusserum mixtures with serum obtained from normal individuals were injected into mice and served as controls.

In all the records of neutralization experiments the nominator represents the number of mice who have survived after inoculation of the virus-serum mixture; the total number of mice involved in the experiment is represented by the denominator.

Table I.-Neutralization of Virus “Ef," by Sera of Patients Suffering from Encephalomyelitis

\begin{tabular}{|c|c|c|c|c|c|c|c|}
\hline \multirow{2}{*}{ No. } & \multirow{2}{*}{ Name } & \multicolumn{5}{|c|}{ Dilution of the virus } & \multirow{2}{*}{ I.N.* } \\
\hline & & $10-2$ & $10-3$ & $10-4$ & $10-5$ & $10-6$ & \\
\hline $\begin{array}{l}1 \\
2 \\
3 \\
4 \\
5 \\
6 \\
7\end{array}$ & $\begin{array}{l}\text { Pe. } \\
\text { Ri. } \\
\text { Vo. } \\
\text { Gu. } \\
\text { Sev. } \\
\text { Mur. } \\
\text { Control with } \\
\text { the serum } \\
\text { of a normal } \\
\text { individual }\end{array}$ & $\begin{array}{l}1 / 4 \\
0 / 4 \\
0 / 4 \\
0 / 4 \\
2 / 4 \\
0 / 4 \\
\\
\\
\\
0 / 4\end{array}$ & $\begin{array}{l}1 / 4 \\
4 / 4 \\
1 / 4 \\
2 / 4 \\
3 / 4 \\
1 / 4\end{array}$ & $\begin{array}{l}4 / 4 \\
1 / 4 \\
2 / 4 \\
3 / 4 \\
4 / 4 \\
2 / 4\end{array}$ & $\begin{array}{l}4 / 4 \\
4 / 4 \\
4 / 4 \\
4 / 4 \\
3 / 4 \\
4 / 4\end{array}$ & $\begin{array}{l}4 / 4 \\
4 / 4 \\
4 / 4 \\
4 / 4 \\
3 / 4 \\
4 / 4\end{array}$ & $\begin{array}{r}400 \\
1,000 \\
100 \\
850 \\
1,350 \\
100\end{array}$ \\
\hline
\end{tabular}

* I.N. $=$ Index of neutralization evaluation of the serum neutralizing activity after the Reed and Muench method (1938).

Table II.-Neutralization of Virus " Sv." by Sera of Patients Suffering from ENCEPHALOMYelitis

\begin{tabular}{|c|c|c|c|c|c|c|c|}
\hline \multirow{2}{*}{ No. } & \multirow{2}{*}{ Name } & \multicolumn{5}{|c|}{ Dilution of the virus } & \multirow{2}{*}{ I.N. } \\
\hline & & $10-2$ & $10-3$ & $10-4$ & $10-5$ & $10-6$ & \\
\hline $\begin{array}{l}1 \\
2 \\
3 \\
4 \\
5 \\
6 \\
7 \\
8 \\
9\end{array}$ & $\begin{array}{l}\text { Ko. } \\
\text { Kal. } \\
\text { Sv. } \\
\text { Va. } \\
\text { Ri. } \\
\text { Ch. } \\
\text { Pe. } \\
\text { Va. } \\
\text { Control with } \\
\text { the serum } \\
\text { of a normal } \\
\text { individual }\end{array}$ & $\begin{array}{l}2 / 4 \\
0 / 4 \\
0 / 4 \\
2 / 4 \\
0 / 4 \\
0 / 4 \\
0 / 4 \\
0 / 4 \\
\\
\\
\\
0 / 4\end{array}$ & $\begin{array}{l}3 / 4 \\
1 / 4 \\
0 / 4 \\
4 / 4 \\
0 / 4 \\
1 / 4 \\
0 / 4 \\
1 / 4\end{array}$ & $\begin{array}{l}3 / 4 \\
3 / 4 \\
1 / 4 \\
4 / 4 \\
3 / 4 \\
1 / 4 \\
3 / 4 \\
2 / 4\end{array}$ & $\begin{array}{l}3 / 4 \\
4 / 4 \\
4 / 4 \\
4 / 4 \\
4 / 4 \\
4 / 4 \\
4 / 4 \\
2 / 4\end{array}$ & $\begin{array}{l}4 / 4 \\
4 / 4 \\
4 / 4 \\
4 / 4 \\
4 / 4 \\
4 / 4 \\
4 / 4 \\
4 / 4\end{array}$ & $\begin{array}{r}12,880 \\
794 \\
117 \\
25,120 \\
550 \\
173 \\
550 \\
25\end{array}$ \\
\hline
\end{tabular}

As seen from these tables, the strains "Sv." and "Ef." may be neutralized by the serum of indi- viduals having suffered from acute disseminated encephalomyelitis. These serological findings clearly show the important role of the viruses we have isolated in the ætiology of acute disseminated encephalomyelitis.

The similarity of morphological changes encountered in acute disseminated encephalomyelitis 0 to those of multiple sclerosis, and experimental encephalomyelitis in animals inoculated with "Sv." ? and "Ef." viruses led us to the suggestion that these $\stackrel{0}{\rightleftarrows}$ viruses might possibly play an important part in? the ætiology, not only of acute encephalomyelitis, but $\overrightarrow{\vec{c}}$ of multiple sclerosis. In the course of our work we endeavoured, though unsuccessfully, to carry out virus investigation of chronic multiple sclerosis. $\frac{a}{\omega}$ The attempt to isolate a virus from the patient's $\frac{\vec{\sigma}}{\vec{\sigma}}$ blood and cerebrospinal fluid proved negative. We $\cong$ had at our disposal three autopsies of patients with on multiple sclerosis. Though every part of the central $\overrightarrow{0}$ nervous system was used in investigations to detect the presence of virus, all proved unsuccessful. In $\vec{\omega}$ this respect we were no more fortunate than in previous investigations.

\section{Relation of Encephalomyelitis to Multiple Sclerosis}

Having at our disposal new possibilities, namely, viruses isolated from patients with acute encephale myelitis, we decided to establish their possib\& relation to multiple sclerosis by means of ser $\vec{P}$ logical tests. For this purpose we attempted neutralize the strains "Sv." and "Ef." with serum collected from patients suffering from chronic multiple sclerosis. Using the same method, we investigated a total of 50 samples of serum obtained from cases of multiple sclerosis, some on more than one occasion. Records of some experiments are given below.

TABle III.-NeUtralization OF THE Virus "Sv." By Sera OF Patients Suffering From Multiple SCLEROSIS

\begin{tabular}{|c|c|c|c|c|c|c|c|}
\hline \multirow{2}{*}{ No. } & \multirow{2}{*}{ Name } & \multicolumn{5}{|c|}{ Dilution of the virus } & \multirow{2}{*}{ I.N. } \\
\hline & & $10-2$ & $10-3$ & $10-4$ & $10-5$ & $10-6$ & \\
\hline $\begin{array}{l}1 \\
2 \\
3 \\
4 \\
5\end{array}$ & $\begin{array}{l}\text { Schk. } \\
\text { Ej. } \\
\text { Sa. } \\
\text { Dol. } \\
\text { Normal se- }\end{array}$ & $\begin{array}{l}3 / 3 \\
0 / 3 \\
0 / 3 \\
0 / 3\end{array}$ & $\begin{array}{l}3 / 3 \\
2 / 3 \\
2 / 3 \\
1 / 3\end{array}$ & $\begin{array}{l}2 / 3 \\
3 / 3 \\
3 / 3 \\
2 / 3\end{array}$ & $\begin{array}{l}2 / 3 \\
3 / 3 \\
3 / 3 \\
3 / 3\end{array}$ & $\begin{array}{l}3 / 3 \\
3 / 3 \\
3 / 3 \\
3 / 3\end{array}$ & $\begin{array}{r}10,000 \mathrm{D} \\
1,623 \text { 을 } \\
1,623 \text { 品 } \\
316\end{array}$ \\
\hline $\begin{array}{r}6 \\
7 \\
8 \\
9 \\
10 \\
11 \\
12\end{array}$ & $\begin{array}{l}\text { rum used } \\
\text { as control } \\
\text { Vo. } \\
\text { Ki. } \\
\text { Dr. } \\
\text { Vi. } \\
\text { Sa. } \\
\text { Mia. } \\
\text { Normal se- } \\
\text { rum used } \\
\text { as control }\end{array}$ & $\begin{array}{l}0 / 3 \\
0 / 4 \\
1 / 4 \\
0 / 4 \\
1 / 4 \\
1 / 4 \\
0 / 4\end{array}$ & $\begin{array}{l}0 / 3 \\
3 / 4 \\
4 / 4 \\
1 / 4 \\
4 / 4 \\
1 / 4 \\
0 / 4\end{array}$ & $\begin{array}{l}0 / 3 \\
4 / 4 \\
3 / 4 \\
4 / 4 \\
3 / 4 \\
4 / 4 \\
3 / 4\end{array}$ & $\begin{array}{l}1 / 3 \\
3 / 4 \\
4 / 4 \\
2 / 4 \\
4 / 4 \\
4 / 4 \\
4 / 4\end{array}$ & $\begin{array}{l}0 / 3 \\
3 / 4 \\
3 / 4 \\
4 / 4 \\
4 / 4 \\
4 / 4 \\
4 / 4\end{array}$ & $\begin{array}{r}1,445 \\
3,063 \\
3,0630 \\
5,000 \AA \\
1,000 \\
316\end{array}$ \\
\hline
\end{tabular}


Table IV.-Neutralization of THE Virus “EF." BY Sera of Patients Suffering from Multiple SCLEROSIS

\begin{tabular}{|c|c|c|c|c|c|c|c|}
\hline \multirow{2}{*}{ No. } & \multirow{2}{*}{ Name } & \multicolumn{5}{|c|}{ Dilution of the virus } & \multirow{2}{*}{ I.N. } \\
\hline & & $10-2$ & $10-3$ & $10-4$ & $10-5$ & $10-6$ & \\
\hline $\begin{array}{r}7 \\
8 \\
9 \\
10 \\
11 \\
12 \\
13 \\
14\end{array}$ & $\begin{array}{l}\text { Schk. } \\
\text { Ej. } \\
\mathrm{Mi} . \\
\mathrm{Fi} . \\
\text { Go. } \\
\text { Normal se- } \\
\text { rum used } \\
\text { as control } \\
\text { Sa. } \\
\mathrm{Ku} . \\
\mathrm{Scha} . \\
\mathrm{Se} . \\
\mathrm{Me} . \\
\text { Eg. } \\
\text { Vo. } \\
\text { Normal se- } \\
\text { rum used } \\
\text { as control }\end{array}$ & $\begin{array}{l}0 / 3 \\
1 / 4 \\
0 / 4 \\
0 / 4 \\
0 / 4 \\
0 / 4 \\
0 / 4 \\
3 / 4\end{array}$ & $\begin{array}{l}0 / 3 \\
4 / 4 \\
0 / 4 \\
3 / 4 \\
3 / 4 \\
2 / 4 \\
0 / 4 \\
3 / 4\end{array}$ & $\begin{array}{l}1 / 3 \\
4 / 4 \\
3 / 4 \\
4 / 4 \\
2 / 4 \\
2 / 4 \\
4 / 4 \\
3 / 4\end{array}$ & $\begin{array}{l}0 / 3 \\
3 / 4 \\
3 / 4 \\
4 / 4 \\
4 / 4 \\
3 / 4 \\
4 / 4 \\
4 / 4\end{array}$ & $\begin{array}{l}1 / 3 \\
4 / 4 \\
4 / 4 \\
4 / 4 \\
4 / 4 \\
3 / 4 \\
4 / 4 \\
4 / 4\end{array}$ & $\begin{array}{r}- \\
3,390 \\
148 \\
2,000 \\
1,000 \\
100 \\
316 \\
10,000\end{array}$ \\
\hline
\end{tabular}

As seen from Tables I, II, III, and IV, both strains "Sv." and "Ef." may be neutralized, not only by sera of patients suffering from acute encephalomyelitis, but also by that obtained from cases of chronic multiple sclerosis. Analogous experiments of neutralization were carried out with sera of patients suffering from other diseases of the nervous system in order to test the specificity of this phenomenon.

Table V.-Neutralization of the Virus "Sv." By Sera of Patients Suffering from Various Diseases OF THE Nervous System and Sera of Healthy ADULTS

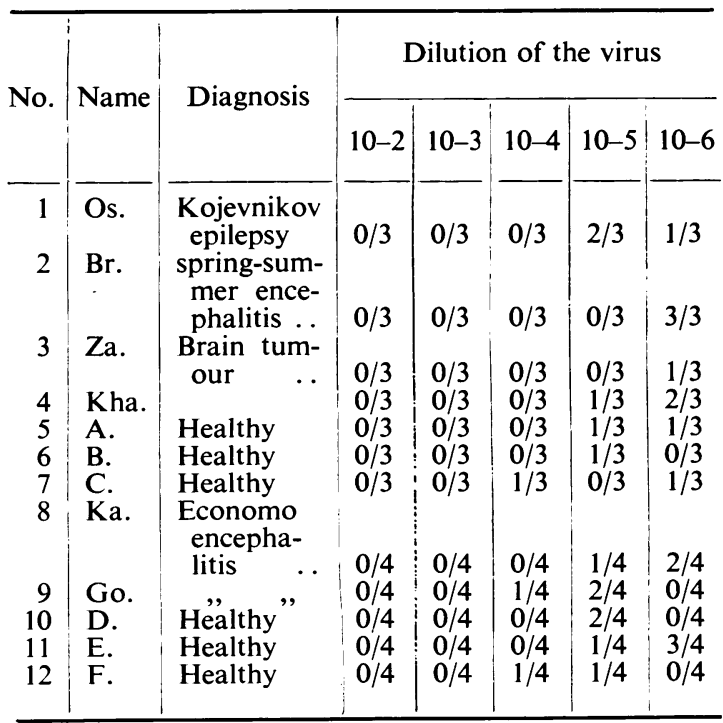

These tests of neutralization showed that antibodies neutralizing the virus of "Sv." and "Ef." strains were present in the serum of a large number of patients suffering from acute disseminated encephalomyelitis and multiple sclerosis, but no such neutralization could be demonstrated using the blood from healthy individuals or from that of patients suffering from other diseases of the nervous system. Data on the content of virus neutralizing antibodies in patients and healthy individuals are given in Table VI.

Table VI.-Total Number of Patients Subjected to SEROlOGICAL EXAMINATION

\begin{tabular}{l|c|c|c}
\hline Diagnosis & $\begin{array}{c}\text { Number of } \\
\text { individuals } \\
\text { investigated }\end{array}$ & \multicolumn{2}{|c}{ Positive results } \\
\cline { 3 - 4 } & 107 & - & -10 \\
\hline $\begin{array}{l}\text { 1. Total number .. } \\
\text { 2. Multiple sclerosis }\end{array}$ & 50 & 25 & 50 \\
3. Acute encepha- & 20 & 14 & 70 \\
$\begin{array}{c}\text { litis . } \\
\text { 4. Other diseases of } \\
\text { nervous system }\end{array}$ & 27 & $1 *$ & $3 \cdot 7$ \\
\hline
\end{tabular}

* The serum which proved positive was obtained from a patient suffering from a disease diagnosed as a vestibular form of encephalitis.

The above findings allow the conclusion that the strains "Sv." and "Ef." isolated from cases of acute encephalomyelitis are able to produce in inoculated animals pathological changes in the nervous system closely resembling those of acute disseminated encephalomyelitis and multiple sclerosis. These virus strains may be neutralized by sera obtained from patients with these diseases. This latter ability provides evidence of specificity of the virus in respect of acute encephalomyelitis and multiple sclerosis.

\section{Comparison with Herpes Virus}

When comparing the strains "Sv." and "Ef." with other viruses, we were mostly concerned with the herpes virus. We do not intend to discuss in detail the possible part this virus plays in the ætiology of epidemic encephalitis; but the fact that the virus of herpes may remain for several years in the body of some of the healthy population without showing any symptoms whatever, must be taken into consideration. In cases where the body is weakened by some kind of infection, the presence of the latent virus is revealed by the occurrence of a herpetic rash on the mucous membranes. This rash may also be promoted in carriers by means of artificial irritants. Two cases of fatal herpetic infection have been described recently in medical literature. Smith, Lennette, and Reames (1941) discovered herpetic inclusions in the brain of a child who succumbed to encephalitis; they produced herpetic infection in mice by inoculation of cerebral substance. Zarafonetis, Smadel, et al. (1944) also detected in the brain of a patient who died from 
Table ViI.-Neutralization of the Herpes Virus, of those of Spring-Summer Encephalitis and of the "Sv." and "Ef." Of Strains by the Immune Sera of Rabbits

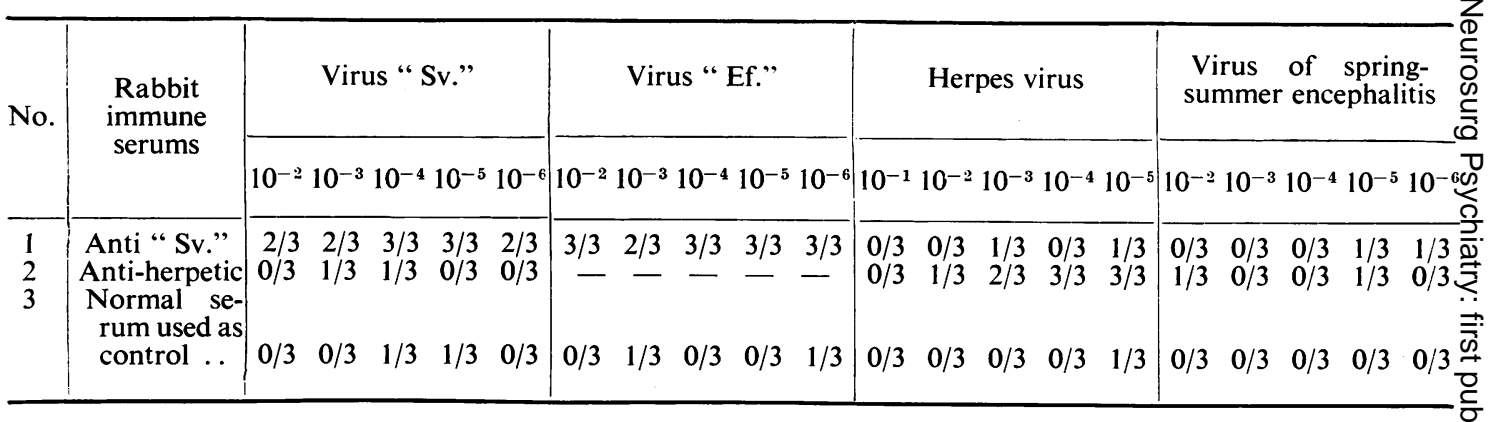

encephalitis acidophil inclusions very much like those seen in herpes infection. The nature of the virus was proved by inoculation of the patient's cerebral substance into animals.

For the purpose of comparative investigation we isolated strains of herpes virus from three patients and selected the most virulent in respect of its neurotropism in rabbits. The difference between herpes strain and the "Sv." and "Ef." experiments was clearly manifested in the first experiments of inoculation of rabbits. We never saw keratitis in rabbits following contamination of the scarified cornea with the "Sv." and "Ef." strains. Simultaneous experiments with herpes virus regularly elicited keratitis. Herpetic inclusions were detected in the cornea after inoculation with the latter virus, while with the "Sv." and " Ef." strains no inclusions were found. We have been unable to demonstrate demyelination in rabbits with herpetic encephalitis; on the other hand, this feature has been characteristic of experimental encephalomyelitis brought about by both "Sv." and "Ef." viruses. Experiments have been made in an attempt to identify the herpes virus by means of neutralization with the sera of patients suffering from multiple sclerosis, sera which seemed to be very active as regards the "Sv." virus. These experiments proved negative. In addition, specific sera obtained from rabbits immunized by the "Sv." strain and herpes virus were prepared. Cross experiments of neutralization of rabbit immune serums were also carried out. The virus of tick-borne spring-summer encephalitis of Far East Siberia was used as control. The results of neutralization of the herpes virus, of those of spring-summer encephalitis, and the "Sv." and " Ef." strains is given in Table VII.

These neutralization experiments confirmed our findings upon the difference between the herpes virus and the virus strains which we have isolated. We have demonstrated that the immune serum of virus "Sv." failed to neutralize the virus of springsummer encephalitis, and likewise, the serum of a patient recovering from this disease failed to neutralize the "Sv." virus. The latter seems quite natural, since there is no epidemiological, clinical, or anatomical similarity between the diseases we have described above, and spring-summer or other forms of seasonal encephalitis.
"Sv." and "Ef." Viruses and other Pathogenic Agents

It is worth while to compare "Sv." and " $\mathrm{Ef}$., viruses, isolated by means of inoculating the initial. $\vec{\circ}$ material into mice, with the pathogenic agents of spontaneous neuro-infectious diseases in the same animal. Two mouse neurotropic viruses are known: the Theiler's virus, which is the agent of poliomyelitiso in mice, and the virus of lymphocytic choriomenin- -0 gitis (Traub). Both viruses may be present in a latent condition in apparently healthy mice and their ${ }^{\mathrm{s}}$ presence may be manifested by serial passages, $\stackrel{\circ}{-}$ thus impairing the purity of the experimental ${ }^{-}$ infection. The major distinctive feature differ $\frac{D}{0}$ entiating the strains we have isolated from viruses of spontaneous mouse infections is the differentee in their pathogenicity for various species. It known that rabbits fail to show susceptibility to the virus of choriomeningitis. Guinea-pigs and? rats exhibit non-susceptibility to the Theiler's virus. This feature is sufficient to differentiate clearlya between our strains and those of spontaneouso neuroinfectious diseases in mice.

Methods of Spreading "Sv." and "Ef." Viruses

In order to elucidate the method of spread of the "Sv." and "Ef." viruses in the body, we carried. out the following experiments. Seven rabbits of equal weight $\left(1,500.0 \mathrm{~g}\right.$.) were inoculated with $\mathrm{a}^{\circ}$ 20 per cent. suspension of virus " Sv." into the right sciatic nerve. This nerve was exposed under loca $\vec{b}$ novocaine anæsthesia $(5.00 \mathrm{c} . \mathrm{cm}$. of a 0.5 per cent. solution), and $0.5 \mathrm{c} . \mathrm{cm}$. of a virus " $\mathrm{Sv}$." suspension was injected by means of a very fine needle. The? wound was sutured with silk. One rabbit was killed each day. The two sciatic nerves, the brain, and the cervical and lumbar portions of the spinal cordN were subjected to histological and virus examination The material thus obtained from rabbits was tested as follows. The nervous tissue was repeatedlyc washed with a sterile physiological saline solution,? minced, and injected into albino mice. The tissue was diluted in the proportion of $1: 10$ and $1: 1,000$ 雨 a dilution of $1: 1,000$ and non-diluted blood were injected. Additional control rabbits, inoculated simultaneously with the virus, developed encephalo- $-\frac{T}{\mathbb{D}}$ myelitis on the 7 th day. Virus examination showed? that the virus could readily be demonstrated in the 


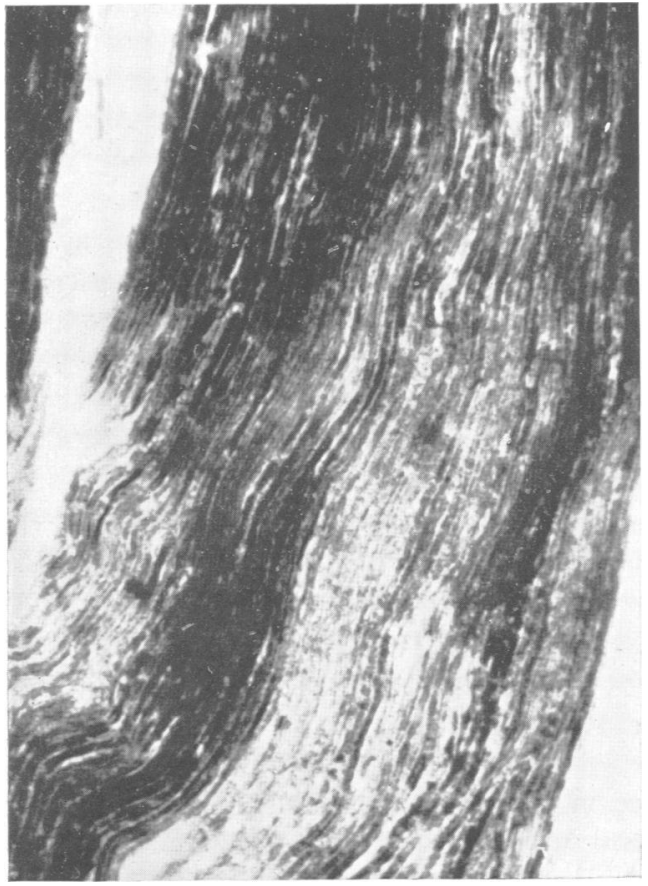

FIG. 8.-Experimental " Sv." encephalomyelitis. Rabbit (N2). Stained after Spielmeyer. Intraneural injection of the "Sv." virus into the right sciatic nerve. Demyelination after 49 hours.

brain the very first day of the experiment; no histological changes in the nervous tissue could be detected, but were found 72 hours later. The presence of virus in the brain the first day following inoculation of a peripheral nerve, and the fact that the brain contained greater amounts of virus than the spinal cord, may be accounted for by a hæmatogenous spread. The level of virus in the blood was not uniform; the virus could readily be detected in the first few days following inoculation of the sciatic nerve, but was absent on the 4th and 5th days. The time of its disappearance coincided with the development of the pathological process in the nervous system. More detailed data on the spread of the virus "Sv." will be the subject of another paper. The most outstanding histological change in the nervous system of rabbits in these series was demyelination of both sciatic nerves, which we could detect 24 hours after inoculation. Both sciatic nerves exhibited disintegration of the myelin sheath in the absence of inflammatory changes. In the first 48 hours this disintegration presented the common picture of degeneration of nerve fibres, and only after having compared such sections with those stained by the method of Bielschowsky could it be established that the axis cylinders showed neither morphological nor quantitative changes. Noticeable fragmentation and destruction of axis cylinders occurred only 72 hours following inoculation. At the same time demyelination became complete. Inflammatory changes in the right sciatic

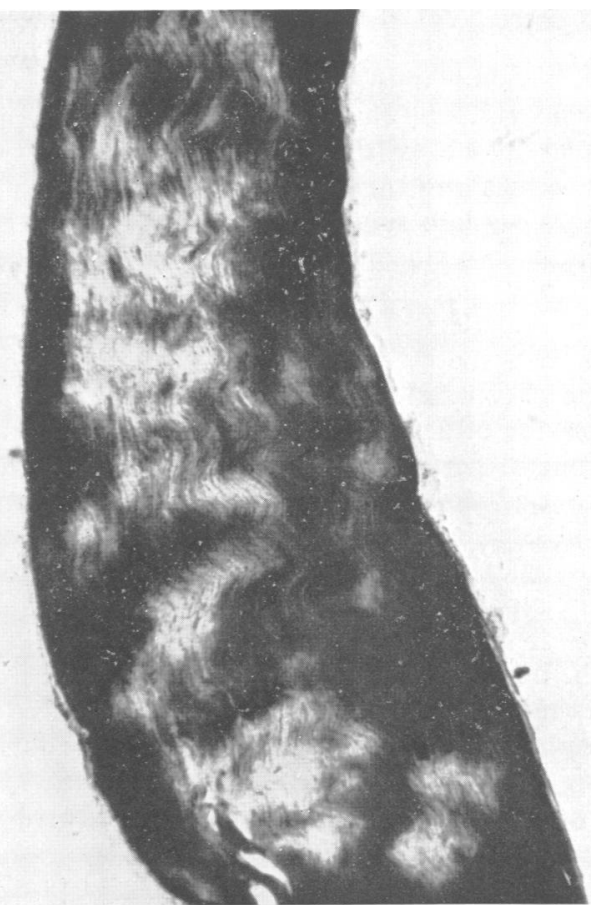

FIG. 9.-Experimental " Sv." encephalomyelitis. Rabbit (N3). Stained after Spielmeyer. Intraneural injection of the "Sv." virus into the right sciatic nerve. Demyelination after 48 hours in the left sciatica nerve.

nerve which had been inoculated were demonstrable at the 48th hour, while inflammatory changes in both nerves could be detected only in the next 24 hours. As to the central nervous system, demyelination appeared later, at the 48th hour after inoculation (Fig. 8) and then in the lumbar spinal cord. Twenty-four hours later demyelination was found in the brain.

The presence of demyelination in both sciatic nerves in the first 24 hours after inoculation, with absence of demyelination in the central nervous system suggests a hæmatogenous spread of the "Sv." virus inoculated into the right sciatic nerve. The foci of necrosis and demyelination scattered throughout the central nervous system may also be related to a hæmatogenous spread of the virus. The greater amount of virus in the brain of infected albino mice as compared with that in the spinal cord is another feature indicating a hæmatogenous spread of the "Sv." virus. The "Sv." virus may also be conveyed by neural paths. Centripetal to the site of inoculation, areas of infiltration were seen in the sciatic nerve. At the same time only small isolated perivascular infiltrative foci were detectable in the lumbar spinal cord. These experiments thus show that the "Sv." virus may follow two paths of spread, the hæmatogenous, and the neural paths. The chief mode of spread is by the blood; spread along neural pathways occurs later and at a slower rate. The initial hæmatogenous spread of the virus in the three forms of encephalomyelitis necessitates 
its passage through the walls of capillaries and eventual fixation in the nervous tissue. The virus traverses not only the capillaries of the central nervous system, but also those of the epidural cellular tissue, whence the virus follows neural pathways along the roots and spaces of the dura, penetrating into subarachnoid spaces.

These experimental findings may be taken as indicating that peripheral nerves are probably the first to be affected following the intraneural inoculation with the "Sv." virus; this spread is by the blood stream. These observations and conclusions are of importance because similar demyelination in peripheral nerves and roots has been observed by us in two cases of acute disseminated encephalomyelitis. The neuro-radicular component of the pathological and clinical picture of disseminated encephalomyelitis has been described by Margulis (1933), and reflects one of the stages of neural spread of the infectious process. In the light of the above experimental data, however, it is believed that changes in peripheral nerves and spinal roots may result from hæmatogenous dissemination. Hence in those cases where the clinical picture of acute disseminated encephalomyelitis presents a longstanding prevalence of the polyneuro-radicular component, it is admissible to suppose that we are dealing with a prolonged epidural stage which is masking the simultaneous lesion of the central nervous system.

\section{Discussion}

Forty years ago, when Marburg (1906) described the acute form of multiple sclerosis, the similarity between the morphological and clinical picture of acute disseminated encephalomyelitis and multiple sclerosis was discussed. Such experienced clinicians as Marburg (1932), Fraenkel and Jakob (1913), and others, had little doubt that acute disseminated encephalomyelitis and acute multiple sclerosis were identical diseases. They realized, however, that it was necessary to establish the nosological uniformity of chronic multiple sclerosis and acute disseminated encephalomyelitis and to prove that the ætiology of both diseases was the same. As Marburg and other authors had no other evidence at their disposal than morphological and clinical findings, their conclusions were far from convincing. In our opinion and in that of a number of authors, morphological discrimination between these two forms is not possible. Thus, Cournand (1930) refused to differentiate acute multiple sclerosis from acute disseminated encephalomyelitis on the basis of the histological picture. Clinical differences to which some authors refer are of little theoretical significance. It has been said that acute encephalomyelitis is accompanied by general cerebral symptoms which fail to occur in acute multiple sclerosis; these symptoms, however, may be encountered, though infrequently, in both forms, especially in cases having a rapid and severe onset, and in which meningeal symptoms are prominent. Febrile reactions may be present in the initial acute stage of both conditions. It is also true that co-ordination of movements is impaired in both diseases. The statement that disorders of sensation bear a different character in acute disseminated encephalomyelitis and acute multiple sclerosis is also in contradiction of the facts. The above data favour the view that acute disseminated encephalomyelitis and acute multiple sclerosis are identical.

The clinical pictures of chronic multiple sclerosis and acute disseminated encephalomyelitis are similar in respect of localization and character of symptoms. It is, therefore, natural for a neuropathologist to hesitate between the diagnosis of acute disseminated encephalomyelitis and chronic multiple sclerosis. In some cases the diagnosis can be established only after further development of the disease. Both diseases have some features in common; namely, wide scattering of the lesions throughout the central nervous system, mostly resulting from lesions in the white matter of both the brain and spinal cord. The similarity of evolution in these two diseases is manifested by the presence of a radicular component in the clinical and pathological picture. This radicular component is suggestive of an epidural stage in both diseases. Differences in the pathological and clinical pictures of acute disseminated encephalomyelitis and chronic multiple sclerosis may depend upon the chronic, or, converse!y, the acute character of the disease, and on the evolution of the pathological process in both cases.

After the outbreak of acute disseminated encephalomyelitis in 1927 and in the years following, Redlich (1927), Flatau (1929), Pette (1929), Maneo kowsky (1930), and others resumed the discussion of the nosological autonomy of acute disseminated encephalomyelitis and its relation to chronic multiple sclerosis. Redlich (1929), Shaefer (1930), and a number of other authors believe that diseases showing occasionally epidemic outbreaks and exhibiting the symptoms of lesions scattered throughout the brain and spinal cord, form a quite independent nosological unity-acute disseminated encephalomyelitis. Other authors, such as Marburg (1942), and Pette (1929), entirely or partially deny the autonomy of this disease. Pette, in several communications (1927-28), advocated the same view and offered some arguments supporting it. He emphasized that, out of Redlich's six patients diagnosed as suffering from acute disseminated encephalomyelitis, two had relapses and were admitted to Nonne's Clinic, where the diagnosis of multiple sclerosis was established. Pette declared that in the future there certainly would be a " stage of encephalomyelitis considered as multiple sclerosis." The problem of the transition of acute disseminated encephalomyelitis into multiple sclerosis should be separated from that of the identical ætiology of these diseases; even identity of ætiology does not necessarily imply transition of the acute form of the disease into a chronic one. We know of a number of cases of Economo's encephalitis which have never shown transition into Parkinsonism; at the same time acute cases of tick-borne encephalitis seldom develop progressive forms in the Far East. 
whereas in the west the same tick-borne encephalitis frequently takes a chronic course. Finally, no chronic stage has as yet been established for acute poliomyelitis. Thus, acute disseminated encephalomyelitis may fail to show transition into multiple sclerosis, although the ætiology of both diseases is the same. Possibly this transition requires some special conditions which are so far unknown. The question of correlation between acute disseminated encephalomyelitis and multiple sclerosis still remains open to discussion. At present, and in the past, there are two contradictory trends in the literature on the subject. Some authors advocate the recognition of two distinct entities (Redlich, 1929; Spielmeyer, 1925; Steiner, 1933; Futer, 1937; and others) while others believe that a single ætiology is responsible for both diseases. Hence, after 40 years, we face the same dilemma. We are now much better prepared for this discussion, for the agent responsible for acute disseminated encephalomyelitis has been isolated and it is now possible to reproduce this disease experimentally. Having established that the strains of virus which have been isolated by us are neutralized by sera of patients who have suffered from either acute disseminated encephalomyelitis or multiple sclerosis, we have attempted to use a serological method to gather information on the correlation between the two diseases. The serum of patients with acute disseminated encephalomyelitis has neutralized both "Sv." and "Ef." viruses in 70 per cent. of cases, and the serum of patients with multiple sclerosis in 50 per cent. of cases. The similarity of both clinical syndromes, identity of morphological changes, serological data, and evolution of the disease allows us to look upon acute disseminated encephalomyelitis as the acute form of chronic multiple sclerosis in those cases where the serological test has proved positive, e.g. when the virus "Sv." has been neutralized by the patient's serum.

In those cases where the serological tests have proved negative, e.g. when the serum of patients suffering from multiple sclerosis has failed to neutralize the virus "Sv." we may postulate either the presence of another agent responsible for both diseases or that a difference in the antigenic structure of the agent, such as occurs in foot-and-mouth disease and poliomyelitis, alters the character of the serological response. Serological tests have shown that some syndromes which are considered as being due to acute disseminated encephalomyelitis, such as optico-encephalitis or optico-myelitis, persistently yield negative results when attempts are made to neutralize the virus "Sv." with the serum of patients suffering from such conditions. This means that these sera contain no antibodies to the above-mentioned virus. Hence the ætiology of symptoms in acute disseminated encephalomyelitis is not necessarily uniform. For instance, it is impossible to state that sporadic and epidemic cases, manifested by the same syndrome, are identical in respect of ætiology.

The standard methods of clinical discrimination which have been employed to determine whether atypical forms are or are not sporadic cases of acute disseminated encephalomyelitis have proved unsatisfactory. For instance, in some of our cases in which the diagnosis of acute encephalomyelitis has been confirmed by post-mortem findings, polyradicular neuritic symptoms have been prevalent, while medullary and bulbo-spinal symptoms have been less pronounced and have occurred later. In other rarer cases, meningeal symptoms with inflammatory changes in the cerebrospinal fluid are more prominent. In a considerable number of patients vestibular and ataxic symptoms have been noted. Cases have been commonly encountered among our clinical material exhibiting focal symptoms or manifesting mental symptoms such as delirium or mental confusion, the results of more diffuse cerebral changes. In these cases the clinical picture yielded no clear basis for the diagnosis of acute disseminated encephalitis, and it was only following neutralization of the "Sv." and " Ef." viruses by the serum of such patients that the true ætiology could be established. But for the serological reaction, these cases would have been diagnosed as acute polyradiculo-neuritis (Landry's paralysis), Economo's vestibular encephalitis, focal meningo-encephalitis, endogenous mental disease, etc. On the basis of these findings it is obvious that the serological test-neutralization of the "Sv." virus by the patient's serum solves the ætiological problem and is thus of major significance for diagnosis. A positive neutralization test is a valuable diagnostic sign of encephalomyelitis and multiple sclerosis, particularly in atypical cases. However, a negative neutralization test does not necessarily exclude the diagnosis of acute disseminated encephalomyelitis and multiple sclerosis. Another method of diagnosis - the skin test with antigen (inactivated "Sv." virus)-is still under investigation.

The treatment of patients with multiple sclerosis is a thankless task, for so far no efficient method has been elaborated. We have attempted specific treatment of acute disseminated encephalomyelitis and multiple sclerosis by means of a vaccine prepared from the cerebral substance of animals (albino mice and guinea-pigs) inoculated with the "Sv." and "Ef." viruses. The vaccine, inactivated by formalin, has proved sterile and harmless. Gradually increasing doses of this vaccine (from 1 to $5 \mathrm{c.cm}$.) have been injected subcutaneously. This treatment consists of several courses, each of 5 injections given twice weekly, with an interval of 3 weeks between the first and second course and of 2 weeks between the second and third course. Nineteen patients suffering from multiple sclerosis have been subjected to this vaccine therapy. Titration of antibodies in the blood of patients was performed a week after each course.

In the group of cases of multiple sclerosis improvement of objective symptoms has been observed in eleven cases, of which six cases showed considerable amelioration. In eight cases no change in the condition of patients has been recorded, and some of them have even shown aggravation. In this latter 
group were two advanced cases of multiple sclerosis, one showing a rapidly progressive course. In the remaining five, the common progressive form of the disease of moderate severity has been found. Improvement following vaccine therapy has manifested itself by considerable amelioration of the general condition of the patients, with an improvement of the function of the nervous system. The patients have become more lively, and fatigue of the lower extremities on walking has diminished. Objectively, paresis of the lower extremities has become less, movements stronger, ataxic and paretic symptoms in the lower extremities less. Romberg's sign has become less pronounced, and the gait more regular and assured. Vaccine therapy has been followed by improvement of objective and subjective sensory disturbance. In some cases dysuria and intention tremor have decreased. Abnormal reflexes are less marked, and have even entirely disappeared in a number of patients.

Vaccine therapy in encephalomyelitis might be considered as successful. There have been only a small number of cases at our disposal. Four patients have undergone this treatment and have shown great improvement; but further accumulation of material is necessary for conclusive evidence.

\section{Conclusions}

As a result of our experimental, anatomical, and clinical findings, we have been able to draw the following conclusions:

1. This work is the first in which experimental investigation of acute encephalomyelitis and multiple sclerosis has been successfully carried out and the infectious origin of these diseases experimentally and clinically confirmed.

2. Virus investigation of patients suffering from acute disseminated encephalomyelitis and multiple sclerosis has enabled us to isolate two strains of filtrable virus-from the blood of one case, and from the cerebral tissue of another case of acute disseminated encephalomyelitis. These strains have proved to be identical, and differ from other known human neurotropic viruses.

3. The syndrome of acute disseminated encephalomyelitis has been produced in animals by means of inoculation with this virus. The morphological picture obtained has proved identical, particularly in rabbits and puppies, to that found in man suffering from acute disseminated encephalomyelitis.
These experiments are the first in which experimental acute encephalomyelitis has resulted from the inoculation of human material.

4. On the basis of the above data an attempt has been made to neutralize the virus thus isolated with the sera of patients suffering from acute disseminated encephalomyelitis and multiple sclerosis. The virus has been neutralized by sera from 50 per cent. of cases of multiple sclerosis and from 70 per cent. of cases of disseminated encephalomyelitis.

5. These positive neutralization tests suggest that both of these diseases result from the same agent. On the other hand, a negative test does not necessarily indicate a different causative agent. Hence acute disseminated encephalomyelitis and multiple sclerosis have the same ætiology in some of the cases, while in others the same anatomical and clinical syndromes may be elicited by other agents, or possibly by the same agent but with a different antigenic structure.

6. A small number of cases of acute disseminated encephalomyelitis and multiple sclerosis, subjected to specific vaccine therapy, has yielded so far satisfactory results. Clinical improvement has been registered, together with a considerable increase of antibodies in the blood.

7. It is our belief that this work is far from being complete. The results put forward by us may form a basis for further investigation.

\section{REFERENCES}

Cournand, A. (1930). La sclérose en plaques aign Paris. Legrand.

Flatau, E. (1929). Encephale, p. 128, 24, 619.

Fraenkel, M., and Jakob, A. (1913). Zges. Neurol., 14, 565 .

Futer, D.C. (1937). Acute Encephalomyelitis. Moscow. Mankowsky, D. (1930). Sovremennaya Psychonevrologia. No. 6.

Marburg, O. (1906). J. Psych., 27, 213.

- (1932). Ibid., 48, 303.

(1942). J. Mt. Sinai Hosp., N.Y., 9, 640.

Margulis, M. S. (1933). Dtsch Z. Nervenhlk., 131, 125.

Pette, H. (1929). Dtsch. Zlb. Nervenheilk, 110, 221.

Redlich, E. (1929). Dtsch. med. Wschr., 55, 562. (1927). Mschr. Psychiatr., 64, 152.

Reed, L. J., and Muench, H. (1938). Amer. J. Hyg., 27, 493.

Shaefer, R. (1930). Dtsch. Arch. klin. Med., 167, 273.

Smith, M. G., Lennette, E. H., and Reames, H. R. (1941). Amer. J. Path., 17, 55.

Spielmeyer, W. (1925). Arch. Psychiatr., 74, 359.

Steiner, G. (1933). Zbl. Neurol., 68, 289.

Zarafonetis, C. J. D., Smadel, J. E., Adams, J. W., and Haymaker, W. (1944). Amer. J. Path., 20, 429. 